\title{
Hausarzt sein oder nicht sein - die Entscheidung fällt spät
}

Djalali, S ; Tandjung, R

\begin{abstract}
Wann haben Sie sich entschlossen, Hausarzt zu werden? Eine Umfrage unter Facharztabsolventen in Allgemeinmedizin überrascht: Das Studium ist offenbar nicht so entscheidend für die weitere Karriere wie die Weiterbildung.
\end{abstract}

Posted at the Zurich Open Repository and Archive, University of Zurich ZORA URL: https://doi.org/10.5167/uzh-101572

Journal Article

Originally published at:

Djalali, S; Tandjung, R (2014). Hausarzt sein oder nicht sein - die Entscheidung fällt spät. PrimaryCare, $14(21): 345$. 


\section{Hausarzt sein oder nicht sein - die Entscheidung fällt spät}

\begin{abstract}
Wann haben Sie sich entschlossen, Hausarzt zu werden? Eine Umfrage unter Facharztabsolventen in Allgemeinmedizin überrascht: Das Studium ist offenbar nicht so entscheidend für die weitere Karriere wie die Weiterbildung.
\end{abstract}

Um dem Hausärztemangel entgegenzutreten, gibt es zwei Ansatzpunkte: das Medizinstudium und die Gestaltung der Weiterbildung. Doch wie kommen entsprechende Massnahmen an?

Um mehr über die Karriereplanung und die Berufsbilder von Hausärztinnen und Hausärzten zu erfahren, wurde eine Querschnittserhebung unter Facharztabsolventen der Jahre 2000-2010 durchgeführt [1]. Eine Kernfrage war, wann während der Lehrjahre sich die Befragten letztendlich für die Hausarztmedizin entschieden.

\section{Titelträger werden Hausarzt}

Alle 1133 Kollegen, die zwischen 2000 und 2010 den Facharzttitel Allgemeinmedizin verliehen bekamen, erhielten eine Einladung zur Umfrage. 456 Ärzte $(40,6 \%)$ beteiligten sich und beantworteten einen Online-Fragebogen mit 28 Multiple-Choice-Fragen. Die Auswertung ergab, dass die Mehrheit (>85\%) aller FacharztabsolventInnen in der Tat eine Karriere als Hausarzt/-ärztin eingeschlagen hatte resp. innerhalb der nächsten Jahre anstrebte. Nur ein kleiner Teil von rund 2\% gab an, den Titel von vorneherein aus anderen Gründen erworben und sich nie für die Hausarztmedizin interessiert zu haben.

\section{Verloren für die Hausarztmedizin}

Die verbliebenen $12 \%$ gaben an, die Weiterbildung gestartet zu haben, mit dem Ziel Hausarzt zu werden, sich jedoch grösstenteils während der Weiterbildungszeit umentschieden zu haben. Als Grund gaben sie an, mehr Interesse an einem Spezialfach entwickelt zu haben oder schlicht keine Perspektive dafür zu sehen, das Berufsleben als Hausarzt mit einem befriedigenden Privatleben zu vereinbaren. Diese Ergebnisse weisen darauf hin, dass die Gestaltung der Weiterbildung einen wesentlichen Einfluss darauf hat, ob das Interesse an der Hausarztmedizin bei jungen Medizinern in der Weiterbildung geweckt und erhalten bleibt oder verloren geht.

\section{Weiterbildung auf Platz eins}

Diese Schlussfolgerung wird durch weitere Resultate der Studie unterstützt. Die Mehrheit (54,4\%) der Befragten, die nach der Facharztweiterbildung Hausärzte geworden waren, gab an, diesen Entschluss während der Weiterbildung gefasst zu haben. 25\% waren während des Studiums dazu angeregt worden, 15,6\% fanden die Motivation bereits vor dem Studium. Nur ein geringer Anteil (4,8\%) entschied sich erst nach dem Erhalt des Facharzttitels.

\section{Neue Berufsbilder}

Auch Fragen zum aktuellen Arbeitspensum und zur Arbeitsorganisation waren Bestandteil der Umfrage. Dabei zeigte sich ein signifikanter Unterschied zwischen den Berufsbildern von Männern und
Frauen. Frauen waren häufiger als angestellte Ärztinnen statt als selbstständige Praxisbetreiberinnen tätig, arbeiteten häufiger in Gruppen- statt Einzelpraxen und waren häufiger im Teilzeit- statt Vollzeitpensum beschäftigt. Da der Frauenanteil unter Medizinstudenten und -absolventen stetig zunimmt und den Männeranteil übertrifft, ist davon auszugehen, dass die vorherrschende Berufsgestaltung der Hausärztinnen bald das generelle Berufsbild in der Hausarztmedizin bestimmen wird.

\section{Schlussfolgerungen}

Für die zukünftige Gestaltung der Hausarztmedizin lässt sich aus dieser Studie also ableiten, dass Weiterbildungsangebote in Allgemeiner Innerer Medizin hausarztspezifischer werden sollten, um hausarztinteressierte Assistenzärzte «bei der Stange» zu halten und, dass Angebote für Teilzeitanstellungen für die neue Hausarztgeneration attraktiver sind.

Literatur

1 Tandjung R, Senn O, Marty F, Krauss L, Rosemann T, Badertscher N. Career after successful medical board examination in general practice - a cross-sectional survey. Swiss Med Wkly. 2013; doi:10.4414/smw.2013.13839.

\author{
Korrespondenz: \\ Dr. med. Sima Djalali \\ Wissenschaftliche Mitarbeiterin \\ Institut für Hausarztmedizin \\ Universität Zürich \\ Pestalozzistrasse 24 \\ 8091 Zürich \\ sima.djalali[at]usz.ch \\ www.hausarztmedizin.uzh.ch
}

PrimaryResearch - das Fenster zur Forschung In dieser Artikelserie stellen wir Forschungsarbeiten aus dem Institut für Hausarztmedizin der Universität Zürich (IHAMZ) vor. Die Originalarbeiten sind entweder «open access» zugänglich oder beim jeweiligen Autor auf Anfrage erhältlich. Die Ergebnisse geben einen spannenden Einblick in die täglichen Herausforderungen, aber auch die Leistung der Hausarztmedizin. An dieser Stelle ein herzlicher Dank an alle Kolleginnen und Kollegen, die sich an den Projekten beteiligen und die hier präsentierten Ergebnisse erst ermöglicht haben!

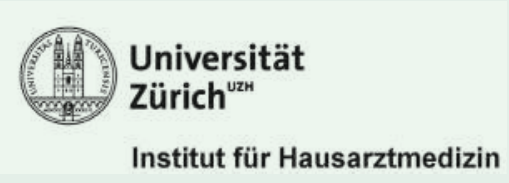

